\title{
Introduction to Special Issue: Use and Misuse of Metal Chelation Therapy
}

\author{
Charles A. McKay, Jr.
}

Published online: 19 November 2013

(C) American College of Medical Toxicology 2013

A 1-day symposium organized by the American College of Medical Toxicology (ACMT) was held at the CDC Conference Center in Chamblee, GA, on February 29, 2012, entitled "Use and misuse of metal chelation therapy" [1]. As physicians who specialize in the evaluation and treatment of patients with concerns about poisoning exposures, medical toxicologists frequently encounter patients who have had esoteric lab testing for environmental exposures or as a component of a workup of poorly defined neuropathies or other symptom complexes. The impetus for this conference was the widespread experience of these physicians with patients who had been treated for chronic symptoms incorrectly attributed to chronic metal toxicity.

During the symposium, experts from a variety of backgrounds spoke to the importance of proper patient evaluation, historic and current use of diagnostic testing, and

This study was previously presented at the conference "Use and misuse of metal chelation therapy" held on February 29, 2012 at the Centers for Disease Control, Atlanta, GA. This conference was jointly sponsored by the American College of Medical Toxicology and the Medical Toxicology Foundation with support from the Agency for Toxic Substances and Disease Registry.

\section{A. McKay Jr. $(\bowtie)$}

Occupational Health Services, Division of Medical Toxicology,

Department of Emergency Medicine, Hartford Hospital,

80 Seymour St., Hartford, CT 06102, USA

e-mail: Charles.McKay@hhchealth.org

C. A. McKay Jr.

Connecticut Poison Control Center,

Department of Traumatology and Emergency Medicine,

University of Connecticut School of Medicine,

263 Farmington Ave., Farmington, CT 06030, USA

C. A. McKay Jr.

American College of Medical Toxicology,

10645 N. Tatum Blvd., Suite 200-111, Phoenix, AZ 85028, USA treatment for metal toxicity, including toxicity considerations for the commonly applied metal chelators. Paul Wax, MD, former president of the ACMT and its current executive director, provided a historical perspective on chelation, including the modern internet promotion of inappropriate chelation treatments.

Carl Herbrandson, $\mathrm{PhD}$, a toxicologist in the Minnesota Department of Public Health, described the questions presented to public health officials by both the public and medical providers regarding concern about environmental exposures and testing and interpretation of tests for metal exposure. He discussed the difficulties that public health officials have in responding to the lay publics' requests for evaluation and concerns about some of the practitioner behavior cited by Dr. Wax.

Charles Lee, MD, senior medical officer within the Center for Drug Evaluation and Research (CDER) of the U.S. Food and Drug Administration (FDA), discussed the role of the Division of New Drugs and Labeling Compliance in attempting to track and counter illegitimate claims for chelating agents and unapproved indications [2].

Richard Wang, DO, a medical toxicologist in the Division of Laboratory Sciences in the National Center for Environmental Health (NCEH) of the Centers for Disease Control and Prevention (CDC), gave an important talk on the definition and derivation of "normal" laboratory test reference ranges. He emphasized factors that are critical to proper laboratory test interpretation, including the important distinction between a result that is "outside a reference range" and a "clinically relevant abnormal" result. In particular, Dr. Wang described the problem with using creatinine adjustment of urine results for children and women, using reference ranges for urinary creatinine excretion developed from adult men.

To further enhance our understanding of some of the important laboratory issues involved in testing for the 
very low concentrations of metals detectable in biologic matrices, Robert Jones, PhD, and Kathleen Caldwell, $\mathrm{PhD}$, both of the Division of Laboratory Sciences of the $\mathrm{NCEH}$, provided descriptions of the instrumentation, quality control procedures, and choice of biologic matrix that are critical for a given question of exposure in order to obtain a relevant, reliable, and reproducible test result. The absence of these careful analytical considerations in many commercial laboratories providing esoteric testing has been demonstrated in the past with hair testing [3] and is likely true of heavy metal testing today.

Michelle Ruha, MD, a member of the board of directors of the ACMT, demonstrated the fallacy of using postchelation challenge measurements of increased excretion of the compounds accessible to chelation as a marker of toxicity, particularly when compared to a non-chelated reference population. In particular, she discussed a simple study she and colleagues conducted, demonstrating the uselessness of one recently popular fad, transdermal DMPS $[4,5]$. She expanded on Dr. Wang's comments regarding creatinine-adjusted results by highlighting the implicit fraud of this method applied to post-challenge urine testing for metals.

Donald Smith, PhD, Professor of Microbiology and Environmental Toxicology at the University of California at Santa Cruz, gave a presentation detailing the physiologic interaction of chelate and chelator (focusing on dimercaptosuccinic acid (DMSA)) and provided data for the efficacy of DMSA treatment of lead poisoning in primates. While early aggressive chelation removed lead from the brain of these animals, ongoing treatment had little effect. In both primate and rodent models, animals that were not lead exposed but given DMSA demonstrated impaired learning and memory similar to the leadexposed animals. These results suggest that there is actual harm from chelation therapy when lead is only present at low concentrations.

Walter Rogan, MD, a pediatrician who was the principal investigator in the National Institutes of Environmental Health Sciences Treatment of Lead-Exposed Children Trial (TLC), reviewed the conduct and findings of that pivotal study [6], which evaluated the impact of prolonged course(s) of DMSA chelation on IQ in more than 700 children with blood lead measurements between 20 and $44 \mu \mathrm{g} / \mathrm{dL}$. Chelation treatment of moderate chronic lead poisoning did not improve intellectual ability or any subtest of neuropsychiatric performance, although blood lead can be decreased by treatment. He discussed the importance of the lead abatement component of the TLC study to decrease ongoing lead exposure.

Mary Jean Brown, $\mathrm{ScD}$, from the Division of Emergency and Environmental Health Services of the NCEH/
CDC discussed the impact of lead on our most vulnerable population, the human in utero. She reviewed CDC's 2010 guidance document regarding the identification and management of lead exposure in pregnant and lactating women [7]. How do recommendations based on occupational or environmental exposure of adults apply to the rapidly developing fetus? Most would agree that some form of a precautionary approach in the face of uncertainty is warranted, but does that approach include consideration of chelation therapy?

Michael Kosnett, MD, MPH, an occupational and environmental medicine physician specializing in medical toxicology at the University of Colorado and a previous president of the ACMT, discussed the historic and current role of chelation therapy in arsenic and mercury poisoning, highlighting the importance of dose and the actual biologic form of the toxin in terms of inducing toxicity as well as the critical element of time to treatment.

Silas Smith, MD, a medical toxicologist at New York University, provided a review of the many other metals and metalloids that, from occupational or unique exposure settings, often need to be addressed by medical practitioners. He provided comments regarding evidence for and against the role of various chelating agents.

In addition, Jeffrey Brent, $\mathrm{MD}, \mathrm{PhD}$, a medical toxicologist at the University of Colorado and previous board member of ACMT, reviewed the complex psychosocial and medical issues evident in the controversy about vaccine safety, focusing on the mercury-containing preservative, thimerosal. Despite the fact that early reports linking thimerosal to pervasive developmental disorder or autism spectrum disorders have not been validated, and the seminal paper by Wakefield has been retracted by the Lancet [8], the controversy continues, reflecting more the polarity of worldviews than it does scientific uncertainty. Perhaps the most telling comment from Dr. Brent's presentation was the red flag that should be raised when proponents conflate dire consequences related to massive exposures to a given element and attribution of serious disease to background levels of exposure in an individual. Combined with Dr. Ruha's warnings about misapplying lab results and using non-appropriate comparison values, these comments form a reasonable basis for assessing the appropriate or inappropriate attribution of disease to these elements.

Dr. McKay, a medical toxicologist at the Hartford Hospital and the University of Connecticut and a member of the executive board of the ACMT, led a panel discussion to bring the key points generated in the day's discussions to the practical question raised by Dr. Herbrandson: How do we as ethical practitioners respond to patients' concerns about their environment, poisoning, and the 
legitimacy of varying claims for diagnosis, testing, treatment, and cure?

We have asked these experts and others to distill their presentations into short papers with sufficient references to provide guidance to the public and clinicians considering heavy metal poisoning. As you read through these presentations, we ask you to consider how the various aspects of exposure, completion of an exposure pathway, patient clinical presentation, careful laboratory testing, and interpretation all impact decisions regarding the use and misuse of metal chelation therapy. An article providing more detail on the analytical issues covered in the symposium by Dr. Wang, Dr. Jones, and Dr. Caldwell should appear in a future issue of the Journal of Medical Toxicology.

Acknowledgment This publication was supported by the cooperative agreement award number 1U61TS000117-04 from the Agency for Toxic Substances and Disease Registry (ATSDR).

Conflict of Interest For the work under consideration for publication, Dr. McKay received a consulting fee/honorarium and reimbursement for travel through the ACMT/ATSDR Cooperative Agreement. Relevant financial activities outside the submitted work included money paid by the state DPH and various private attorney's firms to Dr. McKay and to Dr. McKay's institution for consulting on cases involving chelation issues. Dr. McKay is on the Scientific Advisory Council for the Environmental Health Research Foundation, which addresses issues related to biomonitoring for environmental chemicals.

\section{References}

1. American College of Medical Toxicology (2012) Use and misuse of metal chelation therapy. http:/www.acmt.net/ Library/2012 Chelation_Course/ACMT_Chelation_Course_EVVWD1984.pdf. Accessed 31 Jul 2013

2. Stein R (2010) FDA warns companies promoting products used in chelation procedures, The Washington post. http://www. washingtonpost.com/wp-dyn/content/article/2010/10/14/ AR2010101406962.html. Accessed 30 Jul 2013

3. Seidel S, Kreutzer R, Smith D, McNeel S, Gilliss D (2001) Assessment of commercial laboratories performing hair mineral analysis. JAMA 385(1):67-72

4. College Pharmacy's Center for Advanced Medical Therapeutics TDDMPS ${ }^{\circledR}$. http://www.bowdiges.org/documents/files/Buttar_TDDMPS_Protocol.pdf. Accessed 30 Jul 2013

5. Cohen JP, Ruha AM, Curry SC et al (2013) Plasma and urine dimercaptopropanesulfonate concentrations after dermal application of transdermal DMPS (TD-DMPS). J Med Toxicol 9(1): 9-15

6. Rogan WJ, Dietrich KN, Ware JH, et al. (2001) The effect of chelation therapy with succimer on neuropsychological development in children exposed to lead. NEJM 344(19):1421-1426. http://talkinternational.ca/PDF/chelation-children-lead.pdf. Accessed 30 Jul 2013

7. U.S. Department of Health and Human Services (2010) Guidelines for the identification and management of lead exposure in pregnant and lactating women. http://www.cdc.gov/nceh/lead/publications/ leadandpregnancy2010.pdf. Accessed 30 Jul 2013

8. Editors of the Lancet (2010) Retraction-ileal-lymphoid-nodular hyperplasia, non-specific colitis, and pervasive developmental disorder in children. Lancet 375(9713):445 